\title{
Neonatal cranial ultrasound: current perspectives
}

\author{
This article was published in the following Dove Press journal: \\ Reports in Medical Imaging \\ 26 September 2013 \\ Number of times this article has been viewed
}

\section{Arie Franco \\ Kristopher Neal Lewis \\ Department of Radiology, Medical College of Georgia at Georgia \\ Regents University, Augusta, GA, USA}

Abstract: Ultrasound is the most common imaging tool used in the neonatal intensive care unit. It is portable, readily available, and can be used at bedside. It is the least expensive cross sectional imaging modality and the safest imaging device used in the pediatric population due to its lack of ionizing radiation. There are well established indications for cranial ultrasound in many neonatal patient groups including preterm infants and term infants with birth asphyxia, seizures, congenital infections, etc. Cranial ultrasound is performed with basic grayscale imaging, using a linear array or sector transducer via the anterior fontanel in the coronal and sagittal planes. Additional images can be obtained through the posterior fontanel in preterm newborns. The mastoid fontanel can be used for assessment of the posterior fossa. Doppler images may be obtained for screening of the vascular structures. The normal sonographic neonatal cranial anatomy and normal variants are discussed. The most common pathological findings in preterm newborns, such as germinal matrix-intraventricular hemorrhage and periventricular leukomalacia, are described as well as congenital abnormalities such as holoprosencephaly and agenesis of the corpus callosum. New advances in sonographic equipment enable highresolution and three-dimensional images, which facilitate obtaining very accurate measurements of various anatomic structures such as the ventricles, the corpus callosum, and the cerebellar vermis. Limited studies have been performed to predict that longitudinal measurements of these anatomic structures might predict the clinical outcome of high-risk preterm newborns. Hemodynamic Doppler studies may offer the potential for early intervention and treatment to counter the hazards of developmental delay and a moribund clinical outcome.

Keywords: ultrasound, cranial, neonatal, infants, preterm infants, intracranial hemorrhage, periventricular leukomalacia

\section{Introduction}

Ultrasound is the most widely used cranial imaging modality in the neonatal intensive care unit. Ultrasound machines are portable, the images can be acquired at bedside, and the cumbersome transport of the neonates to the computerized tomography (CT) or the magnetic resonance imaging (MRI) suite is avoided. In addition, ultrasound is considered a safer modality in the pediatric population due to the lack of potential harming effect of ionizing radiation, as in computerized tomography, as well as avoiding the need for sedation frequently required for MRI. Ultrasound is the least costly of all cranial imaging modalities and is readily available in all intensive care units. The modality is operator-dependent and should be performed by an experienced sonographer, neonatologist, or radiologist. In many cases, a final diagnosis and treatment guidance can be achieved with cranial sonography, such as in neonatal germinal matrix hemorrhages. In more complex cases, such as in many congenital malformations,
Correspondence: Arie Franco Department of Radiology, Medical College of Georgia at Georgia Regents University, II 20 I5th Street, Augusta, GA 309I2, USA

Email afranco@gru.edu (c) (i) (5) 2013 Franco and Lewis. This work is published by Dove Medical Press Limited, and licensed under Creative Commons Attribution - Non Commercial (unported, v3.0) License. The full terms of the license are available at http://creativecommons.org/licenses/by-nc/3.0/. Non-commercial uses of the work are permitted without any further permission for the License are administered by Dove Medical Press Limited. Information on how to request permission may be found at: http://www.dovepress.com/permissions.php 
cranial ultrasound serves only as a screening tool that leads to referral for additional, more advanced CT or magnetic resonance images. These advanced imaging tools permit acquisition of images in higher resolution and in different planes that are not available in cranial ultrasound.

\section{Indications for neonatal cranial ultrasound}

Cranial ultrasonography provides important diagnostic information in neonates. The clinical indications for routine scanning in newborn infants are well established. Any change in the normal clinical behavior of the newborn is an indication for cranial ultrasound. Clinical symptoms may range from hypotonic body manifestation to seizures. A low incidence (less than 5\%) of abnormal central nervous system findings was found in the nonasphyxiated term infants with split sutures, cephalohematoma, abnormal neurologic examination, and idiopathic jitteriness. ${ }^{1}$ Routine scanning is suggested in all premature infants as well as term infants with dysmorphic features, macrocephaly, seizure disorders, and in infants with 1- and 5-minute Apgar scores less than 7. Any critically ill preterm infant should receive a cranial ultrasound to search for abnormalities such as hemorrhage, hypoxic-ischemic insult, intrauterine infection, or congenital morphologic abnormalities. Routine screening of severe preterm and low birth weight infants should be performed. In addition, cranial ultrasound examination is the screening technique of choice for assessing preexisting neurological damage in potential neonatal extracorporeal membrane oxygenation (ECMO) candidates. Currently, ultrasound evidence of intracranial hemorrhage greater than grade I in severity is a contraindication to ECMO. ${ }^{2}$ Infants undergoing ECMO are at high risk for brain injury and therefore should undergo serial cranial ultrasound imaging before, during, and after ECMO treatment. ${ }^{3}$

While cranial ultrasound is a very useful imaging modality and generally a first choice for initial imaging, there are limitations. In cases where very detailed brain parenchymal anatomy is needed, MRI provides greater resolution. ${ }^{4}$ Also, in the acute trauma setting, CT is the preferred first line imaging modality. ${ }^{5}$

\section{Technique of cranial ultrasound}

Cranial ultrasound is performed with basic grayscale imaging. For optimal resolution and good overview, a multifrequency $(5-10 \mathrm{MHz})$ linear or convex sector transducer is used. Imaging is obtained through the anterior fontanel in the coronal and sagittal planes. ${ }^{6,7}$ Typically, six to eight coronal images are obtained beginning at the frontal lobes just anterior to the frontal horns and extending to the occipital lobes posterior to the lateral ventricle trigones. ${ }^{8}$ The transducer is then rotated $90^{\circ}$, and approximately five images are obtained, including a midline sagittal view of the corpus callosum and cerebellar vermis in addition to bilateral parasagittal images beginning in the midline and progressing laterally through the peripheral cortex..$^{7-9}$

The mastoid fontanel can be used as an additional port for visualization of the posterior fossa. The mastoid fontanel is located at the junction of the posterior temporal, parietal, and occipital bones and the transducer is positioned approximately $1 \mathrm{~cm}$ posterior to the helix of the ear at a level of approximately $1 \mathrm{~cm}$ above the tragus. Axial images are obtained with the transducer parallel to the orbitomeatal line. By convention, all images are presented with the patient facing left. ${ }^{10}$ Imaging through the posterior fontanel may allow additional views of the occipital lobes. ${ }^{11}$

Doppler images may be obtained for screening vascular structures. ${ }^{9,12}$ The arterial system is assessed for patency and resistance to flow by obtaining a color Doppler image of the circle of Willis. The images are obtained through the anterior fontanel and are used to localize the middle or the internal cerebral artery. Spectral tracings are obtained with peak systolic velocity, end-diastolic velocity, and resistive index. Systolic and diastolic velocities increase with advancing postnatal age and with increasing birth weight regardless of the gestational age. Resistive indices decrease with advancing postnatal age and with increasing birth weight. Any deviation from the normal pattern of the changes in the velocities and the resistive indices may indicate an illness or a pathologic event. The venous system is evaluated for patency of the sagittal sinus and the vein of Galen in the sagittal plane. ${ }^{9}$

\section{Normal sonographic neonatal cranial anatomy}

Knowledge of the normal anatomy is essential for recognizing abnormalities. A general overview of the anatomy and the maturation of the central nervous system can be depicted and correlated with the gestational age. The cortex and the development of the sulci correlate with normal brain maturity. The sulci appear and develop in sequence. The calcarine fissure and most of the anterior part of cingulate sulcus begin to appear before 28 weeks. At 28-31 weeks, all the whole cingulate sulcus and postrolandic sulcus, and most of the superior temporal sulcus and covering of insula, are observable. All of the insular sulci and most of the secondary sulci from the cingulate sulcus appear after 31 weeks 
of gestation. ${ }^{13,14}$ At ultrasound, early sulcal development is best depicted on images obtained perpendicular to the expected course of the sulci. ${ }^{15} \mathrm{~A}$ fissure or sulcus is first seen as a small dot or dimple on the surface of the brain. Later, an obvious V-shaped indentation forms. Finally, the indentation deepens and is visible as a surface notch and an echogenic line that extends into the brain in a Y-shaped configuration. Ultrasound is useful for the evaluation of primary sulci on the medial hemispheric surface (parieto-occipital fissure, calcarine fissure, and cingulate sulcus) and on the lateral convex hemispheric surface (central, post-central, and superior temporal sulci). ${ }^{15-17}$

In sagittal midline plane, the corpus callosum is seen as a sonolucent strip with well-defined echogenic contours overlying the laminae of the septum pellucidum. ${ }^{18}$ In this plane, the vermis of the cerebellum is seen as a very echogenic structure with the fourth ventricle visualized anteriorly (Figure 1). The cisterna magna can be seen inferior to the cerebellum and the aqueduct of Sylvius is seen above the fourth ventricle communicating with the third ventricle.

The frontal horns of the lateral ventricles are seen in the coronal frontal plane (Figure 2). In normal sonograms, the lateral ventricles are symmetric; however, slight asymmetry or slit ventricles may be considered as a normal variant. The ventricles are seen as anechoic structures, due to the cerebrospinal fluid. At about 12 weeks gestational age, the corpus callosum starts to develop from the lamina terminalis as a

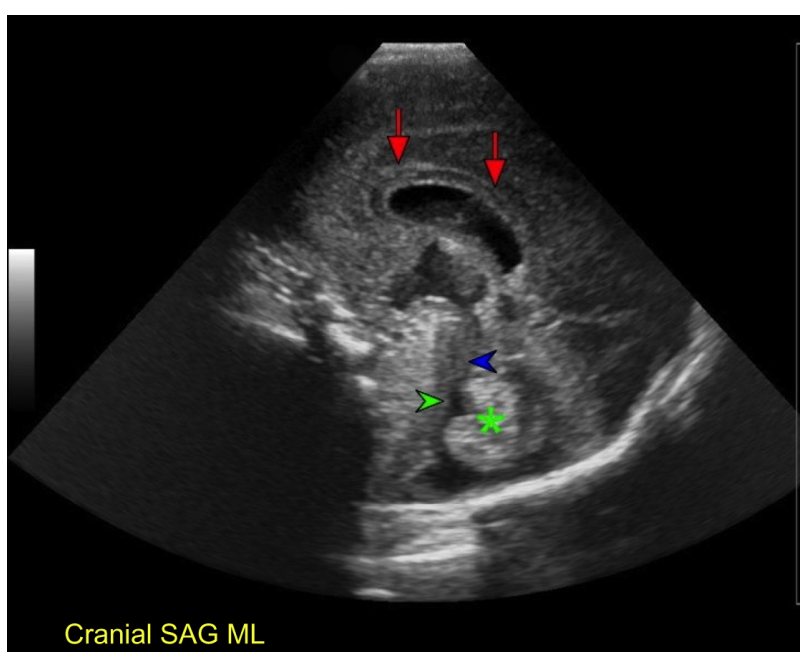

Figure I Normal neonatal brain sonography of a preterm newborn in sagittal midline plane.

Notes: The red arrows point to the corpus callosum, which appears as sonolucent strip with well-defined echogenic contours overlying the cavum septi pelucidi. The vermis of the cerebellum is echogenic (green asterisk). The green arrowhead points to the fourth ventricle and the blue arrowhead points to the quadrigeminal cistern. The absence of the normal sulci of the cortex is indicative of prematurity.

Abbreviation: SAG ML, sagittal midline.

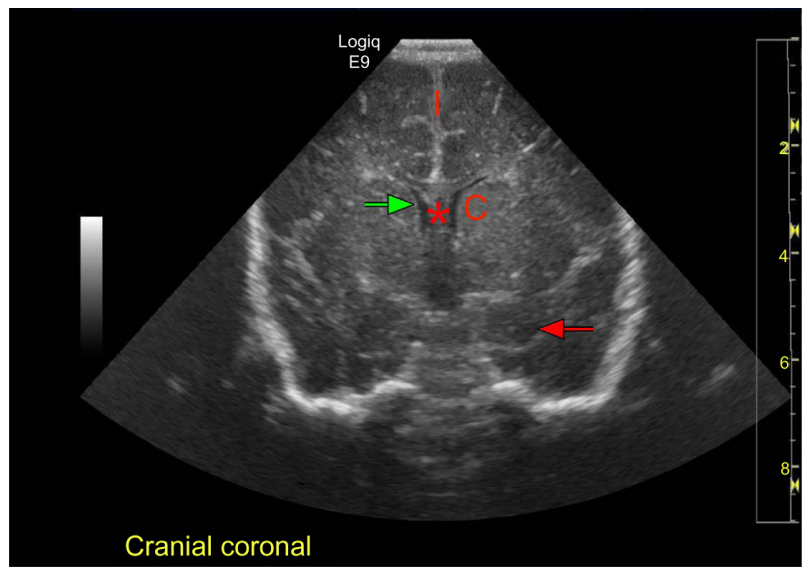

Figure 2 Normal neonatal brain sonography in frontal coronal plane at the level of the frontal lobes.

Notes: Interhemispheric fissure is midline (I). The green arrow points to right anterior horn of the right lateral ventricle. The anterior horn of the lateral ventricle is bounded laterally by the caudate nucleus (C). In severe preterm newborns, an anechoic structure may be seen (not seen in this image), representing the cavum septum pellucidum. When seen, this structure is located between the anterior horns of the lateral ventricles (red asterisk). The red arrow points to the uncus of the temporal horn of the left lateral ventricle.

bundle of fibers that connects the two hemispheres. This is associated with the development of the laminae of the septum pellucidum; two paired, clear membranes. The space between the septa is one cavity anterior to the foramina of Monro, called the cavum septum pellucidum, which is seen as an anechoic structure filled with cerebrospinal fluid, between the anterior horns of the lateral ventricles. ${ }^{19}$ By term, closure of the cavum septum pellucidum has occurred posteriorly in $97 \%$, so that there is generally only a true cavum septum pellucidum at birth; by 3 to 6 months of age, the cavum septum pellucidum is closed in $85 \%$ of infants, ${ }^{20}$ although, in a minority, this cavity remains open until adulthood. ${ }^{21}$ The cavum septum pellucidum is bounded superiorly by a transverse concave sonolucent stripe of the corpus callosum. The anterior horns of the lateral ventricles are bounded laterally by the caudate nucleus.

The choroid plexus is seen in the coronal posterior plane (Figure 3) and in the sagittal lateral plane (Figure 4). The choroid plexus fills the occipital horn of the lateral ventricle. It is a very echogenic structure with smooth homogeneous borders. The choroid plexus tapers towards the caudothalamic groove. In severe preterm newborns, the cavum vergae may be seen between the occipital horns of the lateral ventricles. The cavum vergae is a posterior continuation of the cavum septum pellucidum that closes early in the fetal life. It is less commonly seen in neonates. ${ }^{22}$ The caudothalamic groove is seen in the sagittal lateral view. The groove is located between the caudate nucleus and the thalamus. The floor of the frontal 


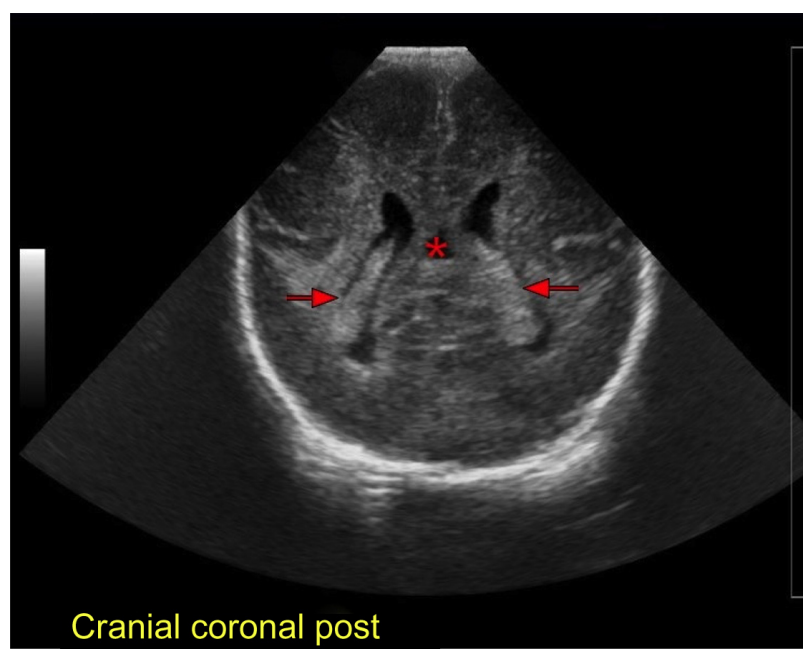

Figure 3 Normal neonatal brain sonography of a preterm newborn in posterior coronal plane at the level of the bodies of the lateral ventricles.

Notes: The red arrows point to the choroid plexus bilaterally, which are echogenic with smooth, homogeneous margins. The red asterisk points to the cavum vergae. Abbreviation: POST, posterior.

horn of the lateral ventricle is bounded by the caudate and the floor of the occipital horn of the lateral ventricle is bounded by the thalamus. The caudothalamic groove demarcates the anteriormost extent of the choroid plexus in the sagittal lateral plane and is an important landmark in identifying germinal matrix hemorrhages. ${ }^{23}$

It is important to recognize the normal variants that should not be mistaken for a significant pathology. Connatal cysts are cysts adjacent to the superolateral margins of the frontal horns of the lateral ventricles. It is believed that they represent

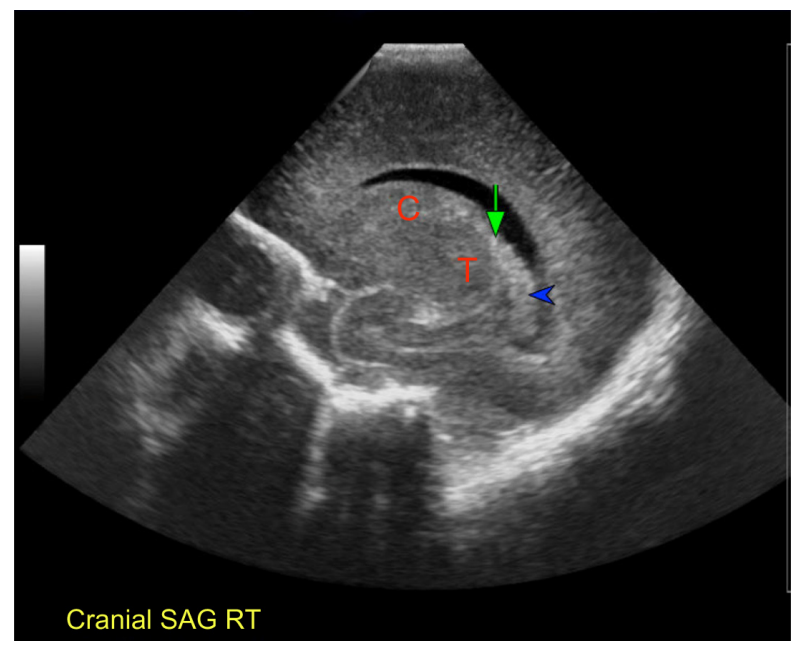

Figure 4 Normal neonatal brain sonography of a preterm newborn in right lateral sagittal plane.

Notes: The green arrow points to the caudothalamic groove. Anterior to the caudothalamic grove is the head of the caudate (C). Posterior to the caudothalamic groove is the thalamus $(T)$. The blue arrowhead points to the choroid plexus that has smooth margins and tapers towards the caudothalamic groove.

Abbreviation: SAG RT, sagittal right lateral. a normal variant. When the ventricle walls are close enough to touch each other, the most external portion of the ventricle acquires a rounded configuration, resulting in sonographic cystic appearance..$^{24,25,39}$ Subependymal cysts could be the result of germinolysis due to hemorrhage, hypoxic-ischemic insult, or neurotropic infection, but can be seen in normal healthy newborns and are considered as normal variants and have a good prognosis. ${ }^{26,39}$ Twenty to $40 \%$ of infants may exhibit asymmetrical ventricular size. ${ }^{27,28}$ A mega cisterna magna (that measures greater than $8 \mathrm{~mm}$ in either sagittal or axial planes) is present in approximately $1 \%$ of infant brains and is a benign finding of no clinical significance. ${ }^{29}$

Rarely, on brain sonography of preterm infants, a hyperechoic focus is seen between the ridges of the interhemispheric region that mimics intracerebral hemorrhage. This hyperechoic focus is a well-recognized artifact and was initially described by Bowerman in $1987 .{ }^{30}$ When a cerebral sulcus or edge of a gyrus is imaged along its long axis, it may appear relatively hyperechoic with respect to adjacent parenchyma, mimicking a focal mass lesion. This "pseudolesion" is most commonly seen on coronal images, when a normal sulcus is imaged tangentially as it courses around the gyrus. Rotation of the transducer to an orthogonal plane can confirm that the brain parenchyma is normal. Other features that suggest a "pseudolesion" are the continuity of the echogenic focus with the normal sulci, and lack of a mass effect. ${ }^{30,31}$ Hyperechoic pseudolesions or periventricular halos may be seen in the white matter. These are due to anisotropic effect and mostly seen in preterm infants. Images obtained at a $90^{\circ}$ angle resolve this finding. Also, these hyperechoic pseudolesions and halos are less echogenic than the adjacent choroid plexus. ${ }^{7,32,33}$ One well documented example is the thalamic pseudolesion, a hyperechoic focus in the thalamus with the appearance of a central thalamic hemorrhage seen when imaging through the posterior fontanel. The hyperechoic focus is no longer seen when imaging through the anterior fontanel. ${ }^{25}$

\section{Common pathologic findings in neonatal cranial ultrasound}

Cerebral intraventricular hemorrhage is the most common central nervous system lesion found in autopsies of newborn infants $\mathrm{s}^{34}$ and the most common finding in ultrasound images performed in the intensive neonatal care unit. Germinal matrix is located in the vicinity of the caudothalamic groove in a fetus. The germinal matrix contains migrating neurons and vessel precursors. It has very thin walled vessels that are sensitive to stress and prone to rupture upon a noxious 
insult leading to hemorrhage. Normally, the germinal matrix involutes by 34 weeks of gestation. A grading system was developed to assess the severity of the hemorrhage. ${ }^{35}$ Grade I consists of hemorrhage confined to the germinal matrix in the subependymal layer (Figure 5). Grade II consists of a germinal matrix hemorrhage that extends to the lateral ventricle without ventricular dilatation (Figure 6). In grade III, hydrocephalus is already present. Grade IV consists of extension of the hemorrhage to the brain parenchyma (Figure 7). Although this classification of Papile in Burstein et $\mathrm{al}^{35}$ is still widely used in interpretation of ultrasound findings, it does not comply with our current understanding of the pathogenesis of the typical periventricular parenchymal lesion. In the new classification of Volpe, ${ }^{36}$ grade IV hemorrhage is due to compression of the terminal vein and not a continuum of the intraventricular hemorrhage as believed by Papile et al. Nowadays, this new classification should be used instead, and parenchymal hemorrhage/venous infarction (grade IV in the Papile et al classification) is recognized as a separate entity. Acute hemorrhage is echogenic on ultrasound images and evolves to an isoechoic and then hypoechoic appearance with time.

Periventricular leukomalacia (PVL) is the major neuropathologic form of brain injury in survivors of premature birth. The pathogenesis of this lesion relates to three major interacting factors. The first two of these, an incomplete state of development of the vascular supply to the cerebral white matter, and a maturation-dependent impairment in regulation of cerebral blood flow underlie a propensity for ischemic injury to cerebral white matter. The third major pathogenetic factor is the maturation-dependent vulnerability of the oligodendroglial precursor cell that

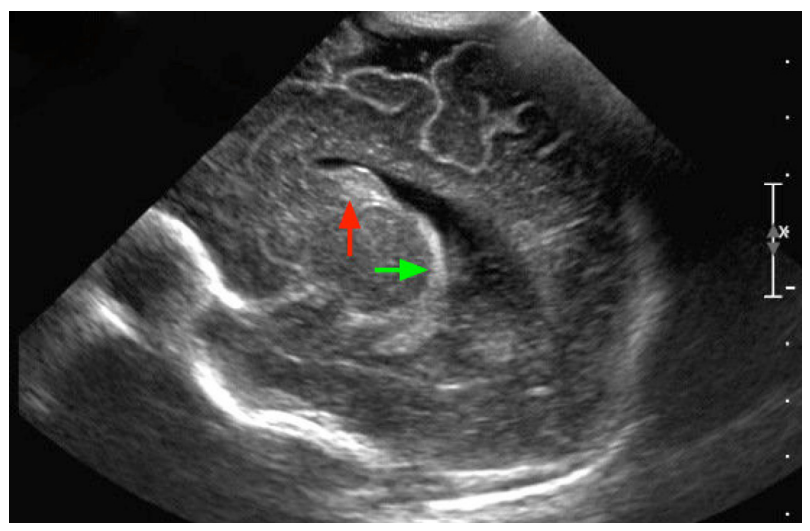

Figure 5 Sagittal right lateral plane of cranial ultrasound.

Notes: Grade I germinal matrix hemorrhage seen as echogenic structure in the subependymal layer, just anterior to the caudothalamic groove (red arrow). The hemorrhage is confined to the subependymal layer with no extension to the lateral ventricle. The normal choroid plexus is echogenic posterior to the caudothalamic groove (green arrow).

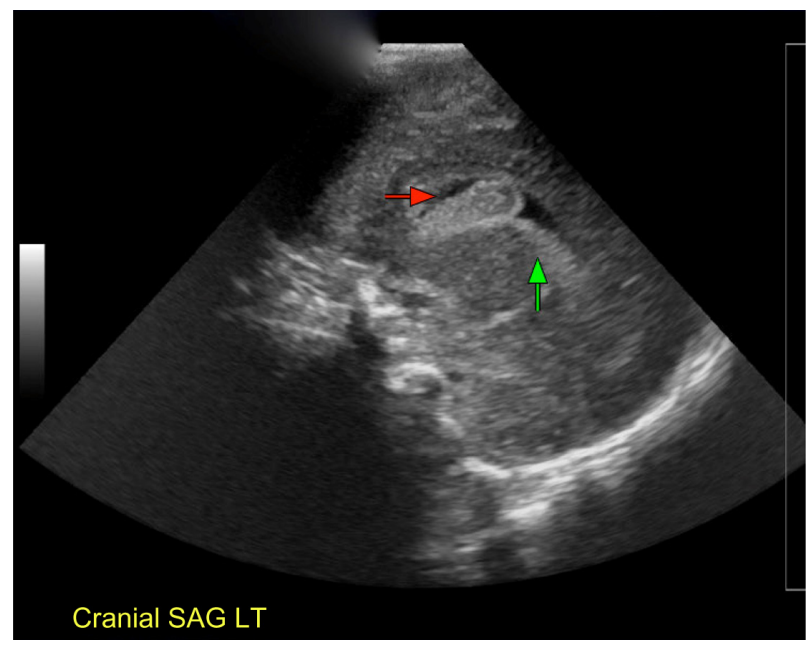

Figure 6 Sagittal left lateral plane of cranial ultrasound.

Notes: Grade II intraventricular hemorrhage seen as echogenic structure inside the lateral ventricle (red arrow). There is no dilatation of the lateral ventricle. The normal choroid plexus is echogenic posterior to the caudothalamic groove (green arrow).

Abbreviation: SAG LT, sagittal left lateral.

represents the major cellular target in PVL. The increased likelihood of PVL in the presence of intraventricular hemorrhage could relate to increases in local iron concentrations derived from the hemorrhage. The important contributory role of maternal/fetal infection or inflammation and cytokines in the pathogenesis of PVL could be related to effects on the cerebral vasculature and cerebral hemodynamics, to generation of reactive oxygen species, or to direct toxic effects on vulnerable oligodendroglial precursors. ${ }^{37}$ The classic necrotic/cystic periventricular leukomalacia is associated with intraventricular hemorrhage. Twenty five percent

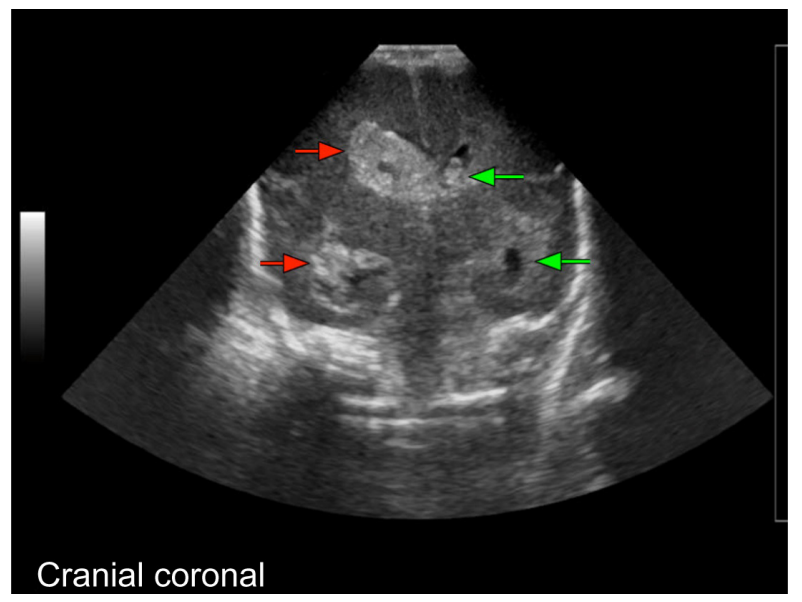

Figure 7 Coronal frontal view of cranial ultrasound.

Notes: Bilateral periventricular hemorrhagic/venous infarction is seen; right (red arrows) more than the left (green arrows). The hemorrhage in the right side exerts mass effect with subfalcine herniation and shift of the interhemispheric sulcus to the left. The hemorrhage is seen in the vicinity of the temporal horns bilaterally. Temporal horns are dilated, indicating hydrocephalus. 
of cases of periventricular leukomalacia become hemorrhagic, especially when associated coagulopathy is present. It was suggested that periventricular hemorrhagic infarction and hemorrhagic leukomalacia may be distinguished by the anatomical location. Hemorrhagic periventricular leukomalacia has a predilection for periventricular arterial border zones, particularly in the region near the trigone of the lateral ventricles. Venous infarction especially in its most hemorrhagic component is prominent more anteriorly. The lesion radiates from the periventricular region at the site of confluence of the medullary and terminal vein and assumes a triangular, fan-shaped appearance in the periventricular white matter. ${ }^{36} \mathrm{~A}$ much more common form of brain injury is noncystic. Transient densities not evolving into cystic lesions may represent a mild degree of leukomalacia when persisting for at least a week. A unilateral parenchymal density may be due to bleeding into an ischemic area, but can also be due to a venous infarction. ${ }^{38}$ The distribution pattern of periventricular leukomalacia as seen in ultrasound is dorsal and lateral to the external angles of the lateral ventricles and involves particularly the centrum semiovale and the optic (trigone and occipital horns) and acoustic (temporal horn) radiations. ${ }^{39,40}$ Periventricular leukomalacia usually occurs in preterm infants of less than 32 weeks gestation. The white matter of these infants is poorly vascularized and contains oligodendrocyte progenitors, which are sensitive to the effects of ischemia and infection. ${ }^{41}$ The cortex is usually spared in preterm infants due to meningeal intra-arterial anastomoses, which involute near term. ${ }^{42}$ Increased echotexture of the periventricular white matter may represent the early sonographic pattern of periventricular leukomalacia. This is a nonspecific finding that must be differentiated from the normal periventricular "blush." Later, a few small cysts appear that represent foci of brain parenchymal loss. The cysts are usually located above the superolateral angle of the anterior horn of the lateral ventricle, at the peritrigonal area and in the periventricular parieto-occipital location (Figure 8). These cysts may coalesce to form a larger cyst, referred to as porencephaly, which is essentially a loss of brain parenchyma (a hole in the brain). This may or may not communicate with the lateral ventricle (Figure 9).

The most commonly reported type of neonatal cerebral arterial infarction in the full-term infant is an ischemic lesion involving the territory of a major cerebral artery. The middle cerebral artery is most commonly affected, and, as found in adult stroke, the left middle cerebral artery is three to four times more frequently involved than the right. Infants with
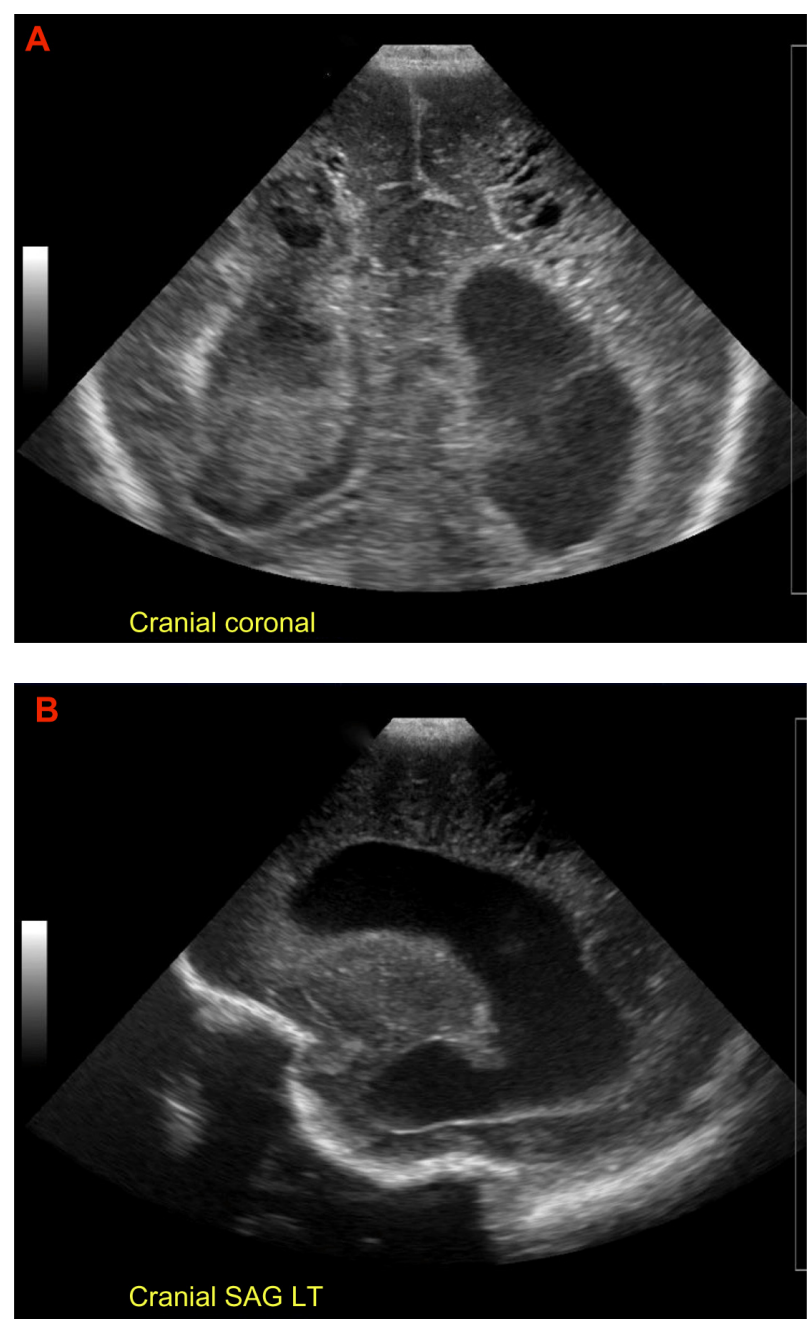

Figure 8 Coronal and left sagittal ultrasound of a preterm newborn.

Notes: Coronal (A) and left sagittal (B) ultrasound of a preterm newborn demonstrates bilateral periventricular hemorrhagic/venous infarction with hydrocephalus. Multiple bilateral tiny cysts are visualized in the periventricular white matter representing periventricular leukomalacia.

Abbreviation: SAG LT, sagittal left lateral.

involvement of the anterior or posterior cerebral artery may be asymptomatic and the abnormality is difficult to detect with cranial ultrasound. ${ }^{43,44}$

Advances in technology, including three-dimensional and high-resolution ultrasound equipment, enables the early diagnosis of congenital malformations. ${ }^{45}$ Holoprosencephaly is a complex brain malformation resulting from incomplete cleavage of the prosencephalon. ${ }^{46}$ This is a severe central nervous system malformation that can be diagnosed early in utero or during the newborn period. Sonographic findings include a monoventricle and absence of the interhemispheric echogenic fissure, fused thalami, hydrocephalus, absence of corpus callosum, and associated craniofacial abnormalities (Figure 10). ${ }^{47,48}$ This entity includes a large spectrum of abnormalities from minor absence of the septum pellucidum 


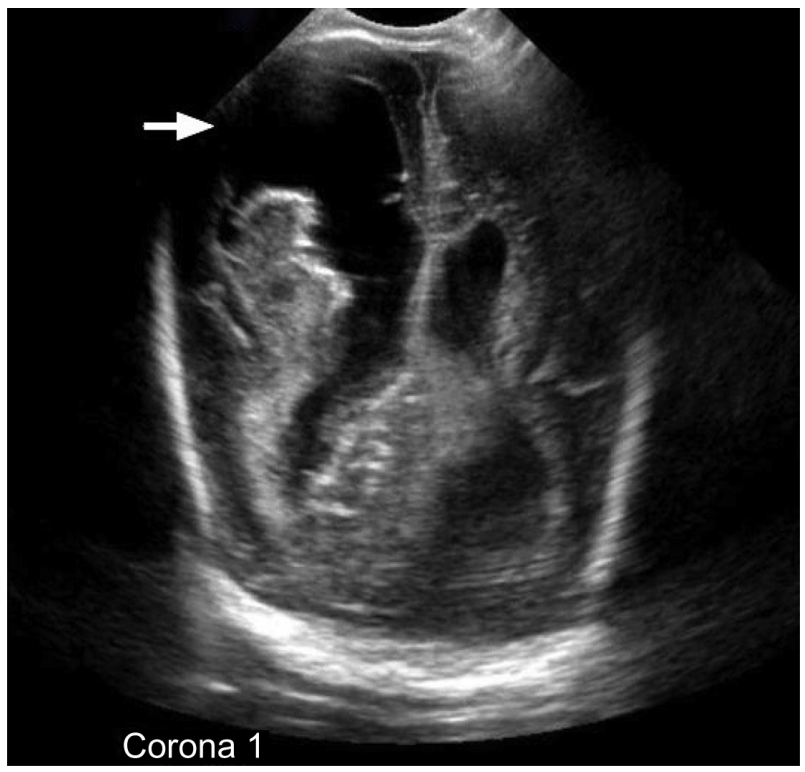

Figure 9 Coronal ultrasound of a preterm infant.

Notes: Demonstrates porencephaly (arrow) that communicates with the right lateral ventricle. The infant had periventricular venous infarction that complicated into cystic periventricular leukomalacia. Early images revealed multiple small cysts that subsequently coalesce to form porencephaly.

(septo-optic dysplasia) (Figure 11) to the severe form, alobar holoprosencephaly.

Absence of the corpus callosum is a congenital malformation that can be diagnosed early by ultrasound. The sonographic findings include partial or complete nonvisualization

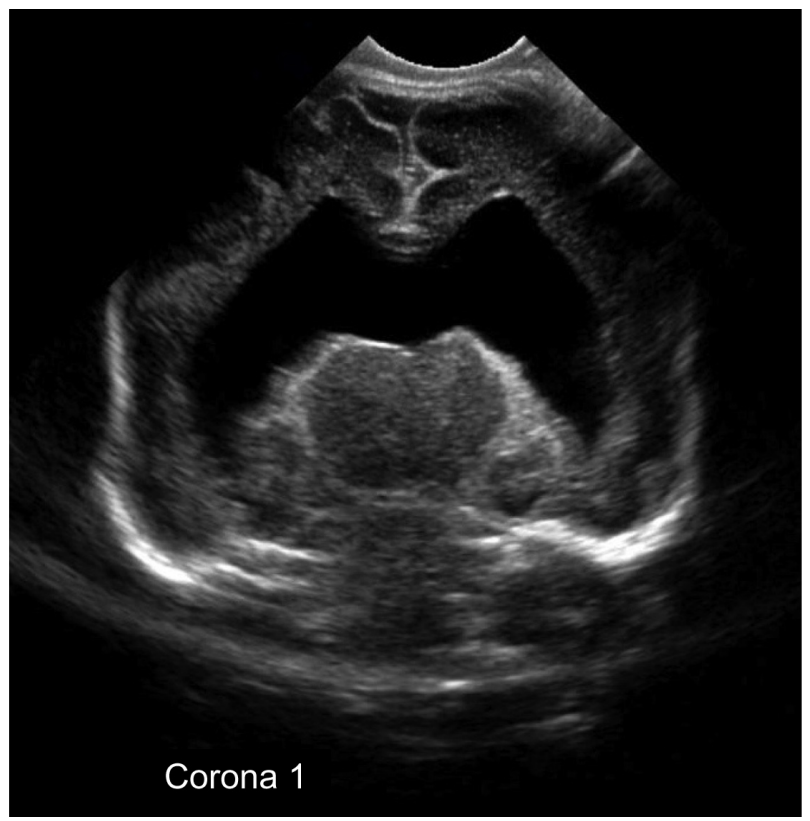

Figure 10 Coronal sonography of a newborn.

Notes: Demonstrates partial absence of the interhemispheric fissure. Monoventricle and fused thalami is seen and there is hydrocephalus. Findings are consistent with semi lobar holoprosencephaly.

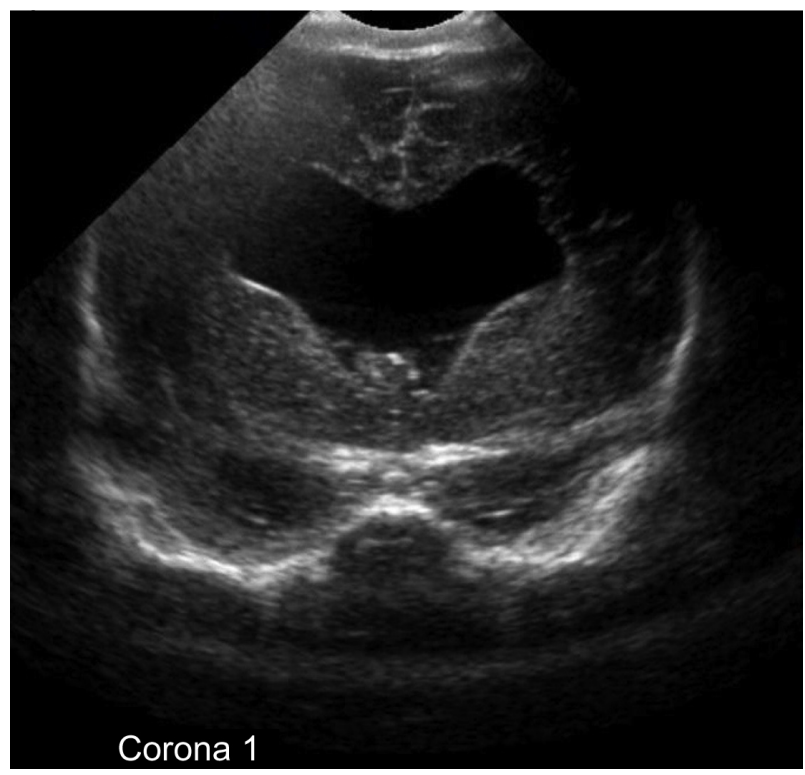

Figure I I Coronal frontal sonography of a preterm newborn.

Notes: Coronal frontal sonography demonstrates absence of the cavum septum pellucidum. The frontal horns of the lateral ventricles are fused. The findings are consistent with septo-optic dysplasia (De Morsier syndrome).

of the midline hypoechoic stripe that represents the corpus callosum. There is separation of the lateral ventricles with increased angulation and concavity of the medial border due to the prominent bundles of Probst, and dilatation and elevation of the third ventricle with interposition between the lateral ventricles. The occipital horns of the lateral ventricles are dilated and parallel to each other, called colpocephaly (Figure 12). ${ }^{49}$

Many other congenital abnormalities can be diagnosed by cranial neonatal ultrasound. Various cystic lesions such as choroid plexus cysts, subependymal cysts, periventricular leukomalacia cystic lesions, and large cyst-like lesions, such as porencephaly, schizencephaly, and arachnoid cysts, can be diagnosed and differentiated with ultrasound..$^{22,39}$ The use of the posterior fontanel and the mastoid fontanel significantly augment the power of the sonographic diagnosis of posterior fossa abnormalities. ${ }^{50}$ Evaluation of the posterior fossa permits transverse measurements of the posterior fossa for evaluation of the gestational age, ${ }^{51}$ screening for abnormalities such as Dandy-Walker syndrome complex, ${ }^{52}$ ArnoldChiari syndrome, mega cisterna magna, and posterior fossa arachnoid cyst. ${ }^{53}$

\section{Current perspectives}

Neonatal cranial ultrasound is routinely performed to screen preterm infants for complications of prematurity. The advance of new techniques, specifically equipment that provides high resolution and three-dimensional images, permits analyzing 

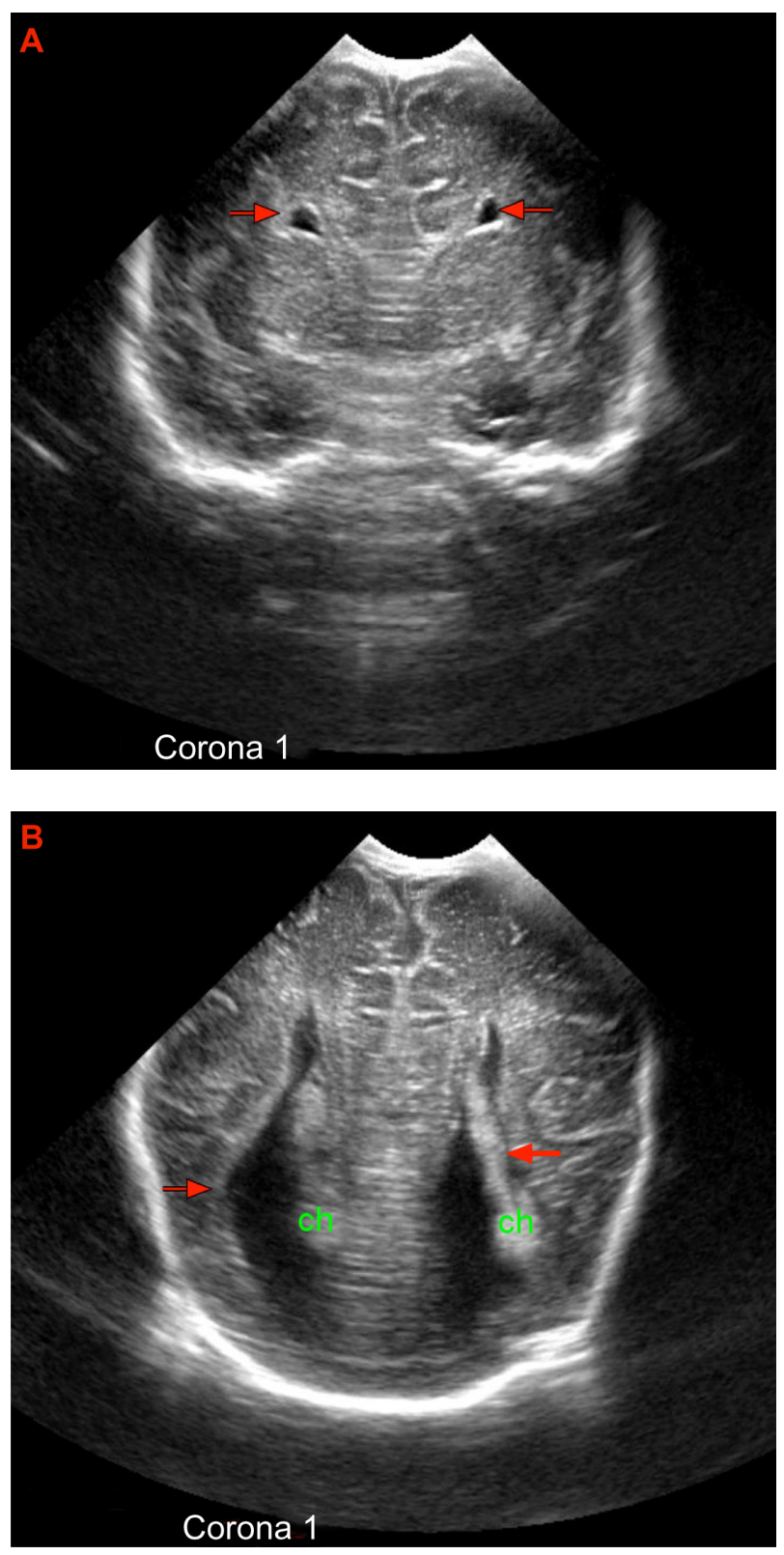

Figure 12 Coronal view of a preterm newborn.

Notes: (A) Coronal frontal view of a preterm newborn demonstrates increased separation between the frontal horns of the lateral ventricles (red arrows). There is increased angulation of the frontal horns and concavity to the medial border. (B) Coronal occipital view of a preterm newborn. The occipital horns of the lateral ventricles (red arrows) are dilated and parallel to each other, consistent with colpocephaly. Findings are consistent with agenesis of the corpus callosum. Abbreviation: ch, normal choroid plexus.

the images in many aspects. Studies were performed to assess the feasibility and the variability of measurements of lateral ventricles. $^{54}$

An automated image processing based approach that measures the anterior horn width as the distance between medial wall and floor of the lateral ventricle at the widest point was developed. Measurement is done in the plane of the scan at the level of the intraventricular foramina. This study was based on neonatal brain ultrasound images in the midline coronal view. In addition to ventriculomegaly detection, this work also included both cross sectional and longitudinal study of anterior horn width of lateral ventricles. ${ }^{55}$ In another study, reference ranges for the measurement of the intracranial ventricles in preterm infants from 23 to 33 weeks gestational age were provided and can be used in the diagnosis and assessment of ventricular enlargement in preterm infants. ${ }^{56}$ Also, longitudinal reference curves were established for the size of the neonatal lateral ventricles between 24 and 42 weeks gestational age, which may allow for early identification and quantification of ventriculomegaly due to either posthemorrhagic ventricular dilation or periventricular white matter loss. ${ }^{57}$

Apart from ventricular measurement, there is an effort to measure other cerebral structures in order to assess the potential developmental abnormalities to enable early intervention and treatment. A study of the growth trajectory of the corpus callosum was performed with cranial ultrasound in very premature infants. A daily measurement of the length of the corpus callosum on cranial ultrasound was performed and found to be reproducible. The study concluded that the neonates with poorer neurodevelopmental outcomes have a shorter corpus callosum at term equivalency. The corpus callosum grows at a much lower rate postnatally than in utero among very premature infants. ${ }^{58}$ Other studies were performed to assess the reliability of linear sonographic cerebellar vermis measurement. Cerebellar growth in late gestation is impeded by prematurity, which may adversely affect neurocognitive development. It was concluded that, with adequate images, linear ultrasound measurements of cerebellar vermis are reliable. ${ }^{59}$ A study was also performed to assign reference values for nasal bone length in the first trimester of pregnancy. ${ }^{60}$

Another study was performed to describe a region of hyperechoic white matter adjacent to the atrium of the lateral ventricle of preterm infants and to speculate on the relevance of detecting preterm white matter injury. One first week standard coronal image was used for measurement of grey values around the para-atrial (the anatomic structure adjacent to the atrium of the lateral ventricle) region of interest relative to the choroid plexus. For verification of the sonographic anatomy, magnetic resonance images of an adult brain were used. For reference, neuroanatomical images were compared in several atlases. The symmetrical and unchanged acoustic character between 26 and 31 weeks of gestational age argues in favor of the hypothesis that the para-atrial region of interest is an anatomical structure. The localization of the hyperechoic 
band supports the hypothesis that it represents part of the optic radiation. ${ }^{61}$

Other studies that consist of measurements of anatomic structures have been performed. In one of these, the subarachnoid space during routine cranial sonography was measured in order to assess an indirect method of monitoring brain growth in preterm infants. It was concluded that the mean subarachnoid space is normally $<3.5 \mathrm{~mm}$ in preterm infants. In this study, the difference between initial and follow up scans suggested reduced brain growth in extrauterine preterm babies. ${ }^{62}$ Another study confirmed the relationship between gestational age and transverse cerebellar diameter. ${ }^{63}$

Doppler ultrasound parameters can serve as a tool for predicting developmental delay. It was found that the leftward hemodynamic status of the middle cerebral arteries, as measured by cranial Doppler ultrasound in the neonatal period, predicts early motor outcome in term infants. ${ }^{64}$ Transcranial Doppler is a sensitive technique for circulatory arrest diagnosis in brain death when patterns such as reverberant flow and short systolic spikes are observed. In infants, the nonossified fontanels compensate for intracranial hypertension. It was found that reverberating flow may be indicative of circulatory arrest even if with a large peak and with a high peak systolic velocity. It was concluded that heavy fontanel compression might reproduce the classical adult transcranial Doppler patterns of brain death, thus supporting the diagnosis of cerebral circulatory arrest. ${ }^{65}$ It has been shown that a pathological prenatal Doppler result is highly predictive for neonatal abnormal cranial ultrasound findings in addition to gestational age and can therefore be used for risk assessment. ${ }^{66}$

Findings in cranial ultrasound were compared to MRI findings. It was found that all severe white matter abnormalities identified on MRI at term age were also detected by cranial ultrasound at term, providing the examinations were performed on the same day. Infants with a normal cranial ultrasound at term age were found to have a normal MRI or only mild white matter abnormalities on MRI at term age. ${ }^{67}$ Comparison of cranial ultrasound and MRI findings were obtained in a group of preterm infants between birth and term. It was concluded that ultrasound accurately predicted the presence of germinal layer hemorrhage, intraventricular hemorrhage, and hemorrhagic parenchymal infarction on MRI. However, its ability to predict the presence of diffuse excessive high-signal intensity and small petechial hemorrhages in the white matter on T2 weighted images is not as good, but improves on scans performed at 7 days or more after birth. In addition, normal white matter echogenicity on cranial ultrasound is not a good predictor of normal white matter signal intensity on MRI. ${ }^{68}$

Another study describes the incidence and evolution of brain imaging findings in very preterm infants (gestational age $<32$ weeks) assessed with sequential cranial ultrasound throughout the neonatal period and MRI around term age. Frequent findings on both cranial ultrasound and MRI around term included ventricular dilatation, widened extracerebral spaces, and decreased cortical complexity. MRI additionally showed punctate white matter lesions and diffuse and excessive high signal intensity, but did not depict lenticulostriate vasculopathy and calcifications and was less reliable for germinolytic and plexus cysts. Cranial ultrasound detected most abnormalities that have been associated with abnormal neurodevelopmental outcome. ${ }^{69}$ Another study compared ultrasound, MRI, and neurodevelopmental outcome in preterm infants and concluded that early MRI provided additional information in those with cystic periventricular leukomalacia. MRI at term age could assess the posterior limb of the internal capsule, which was useful in children with unilateral parenchymal involvement, for prediction of subsequent hemiplegia, and, to a lesser degree, in bilateral cystic periventricular leukomalacia for prediction of diplegia or quadriplegia. ${ }^{4}$ Another study compared the value of serial cranial ultrasound examinations with a single magnetic resonance imaging study before discharge in very low birth weight preterm infants to predict cerebral palsy. As a predictor of outcome for cerebral palsy, MRI at near-term in very low birth weight preterm neonates is superior to ultrasound. However, both cranial ultrasound and MRI demonstrate high specificity. ${ }^{70}$

Major intracranial lesions in the preterm infant should be recognized with sequential cranial ultrasound studies and will predict those with nonambulatory cerebral palsy. Magnetic resonance imaging at term-equivalent age will refine the prediction by assessment of myelination of the posterior limb of the internal capsule. Prediction of motor outcome in preterm infants with subtle white matter injury is difficult, even with conventional MRI. MRI is a better tool to predict outcome in the term infant with hypoxic-ischemic encephalopathy or neonatal stroke. ${ }^{71}$

\section{Conclusion}

Recent advances in ultrasound technology and techniques, such as scanning through the mastoid fontanel and power Doppler and spectral Doppler during fontanel compression, have dramatically improved the ability to image the structure and hemodynamics of blood flow in the neonatal brain. ${ }^{72}$ 
Cranial ultrasound is currently in routine use in neonatal intensive care units and was found to be an excellent and noninvasive tool for brain imaging during the neonatal period. ${ }^{73}$ It enables screening of the brain and serial imaging in highrisk neonates. In experienced hands, cranial ultrasound is an excellent tool to detect the most frequently occurring brain abnormalities in preterm and full-term neonates, to study the evolution of lesions, and to follow brain maturation. ${ }^{74}$ Continuing advances in the development of ultrasound contrast agents, in higher resolution of three-dimensional images, and improved transducers may hold promise for additional improvement in the anatomic and the physiologic diagnostic capabilities of transcranial neonatal ultrasound. This may enable early intervention and treatment and may improve clinical outcome.

\section{Disclosure}

The authors report no conflicts of interest in this work.

\section{References}

1. Sims ME, Halterman G, Jasani N, Vachon L, Wu PY. Indications for routine cranial ultrasound scanning in the nursery. J Clin Ultrasound. 1986;14(6):443-447.

2. von Allmen D, Babcock D, Matsumoto J, et al. The predictive value of head ultrasound in the ECMO candidate. J Pediatr Surg. 1992;27(1): 36-39.

3. Bulas DI, Taylor GA, O’Donnell RM, Short BL, Fitz CR, Vezina G. Intracranial abnormalities in infants treated with extracorporeal membrane oxygenation: update on sonographic and CT findings. AJNR Am J Neuroradiol. 1996;17(2):287-294.

4. Roelants-van Rijn AM, Groenendaal F, Beek FJ, Eken P, van Haastert IC, de Vries LS. Parenchymal brain injury in the preterm infant: comparison of cranial ultrasound, MRI and neurodevelopmental outcome. Neuropediatrics. 2001;32(2):80-89.

5. Fernandy S, Obaldo RE, Walsh IR, Lowe LH. Neuroimaging of nonaccidental head trauma: pitfalls and controversies. Pediatric Radiology. 2008;38(8):827-838.

6. Epelman M, Daneman A, Kellenberger CJ, et al. Neonatal encephalopathy: a prospective comparison of head US and MRI. Pediatr Radiol. 2010;40(10):1640-1650.

7. Seigel M, editor. Pediatric Sonography. 3rd ed. Philadelphia: Lippincott Williams \& Wilkins; 2002.

8. Rumack C, Wilson S, Charboneau J. Diagnostic Ultrasound. St Louis: Mosby; 2005.

9. Lowe LH, Bailey Z. State-of-the-art cranial sonography: Part 1, modern techniques and image interpretation. AJR Am J Roentgenol. 2011;196(5):1028-1033.

10. Buckley KM, Taylor GA, Estroff JA, Barnewolt CE, Share JC, Paltiel HJ. Use of the mastoid fontanelle for improved sonographic visualization of the neonatal midbrain and posterior fossa. AJR Am J Roentgenol. 1997;168(4):1021-1025.

11. Anderson NG, Hay R, Hutchings M, Whitehead M, Darlow B. Posterior fontanelle cranial ultrasound: anatomic and sonographic correlation. Early Hum Dev. 1995;42(2):141-152.

12. North K, Lowe L. Modern head ultrasound: normal anatomy, variants, and pitfalls that may simulate disease. Ultrasound Clin. 2009;4: 497-512.

13. Huang CC. Sonographic cerebral sulcal development in premature newborns. Brain Dev. 1991;13(1):27-31.
14. Murphy NP, Rennie J, Cooke RW. Cranial ultrasound assessment of gestational age in low birthweight infants. Arch Dis Child. 1989;64(4): 569-572.

15. Toi A, Lister WS, Fong KW. How early are fetal cerebral sulci visible at prenatal ultrasound and what is the normal pattern of early fetal sulcal development? Ultrasound Obstet Gynecol. 2004;24:706-715.

16. Monteagudo A, Timor-Tritsch IE. Development of fetal gyri, sulci and fissures: a transvaginal sonographic study. Ultrasound Obstet Gynecol. 1997;9:222-228.

17. Ghai S, Fong KW, Toi A, Chitayat D, Pantazi S, Blaser S. Prenatal US and MR imaging findings of lissencephaly: review of fetal cerebral sulcal development. Radiographics. 2006;26(2):389-405.

18. Plasencia W, Dagklis T, Borenstein M, Csapo B, Nicolaides KH. Assessment of the corpus callosum at 20-24 weeks' gestation by three-dimensional ultrasound examination. Ultrasound Obstet Gynecol. 2007;30(2):169-172.

19. Winter TC, Kennedy AM, Byrne J, Woodward PJ. The cavum septi pellucidi: why is it important? J Ultrasound Med. 2010;29(3):427-444.

20. Shaw CM, Alvord EC Jr. Cava septi pellucidi et vergae: their normal and pathological states. Brain. Mar 1969;92(1):213-223.

21. Rumack $C$, Drose J. Neonatal and infant brain imaging. In: Rumack $C$, Wilson S, Charboneau J, editors. Diagnostic Ultrasound. St Louis: Elsevier; 2005:1623-1702.

22. Mack LA, Rumack CM, Johnson ML. Ultrasound evaluation of cystic intracranial lesions in the neonate. Radiology. 1980;137(2):451-455.

23. Bowie JD, Kirks DR, Rosenberg ER, Clair MR. Caudothalamic groove: value in identification of germinal matrix hemorrhage by sonography in preterm neonates. AJR Am J Roentgenol. 1983;141(6):1317-1320.

24. Rosenfeld DL, Schonfeld SM, Underberg-Davis S. Coarctation of the lateral ventricles: an alternative explanation for subependymal pseudocysts. Pediatr Radiol. 1997;27(12):895-897.

25. Enríquez G, Correa F, Lucaya J, Piqueras J, Aso C, Ortega A. Potential pitfalls in cranial sonography. Pediatr Radiol. 2003;33(2):110-117.

26. Makhoul IR, Zmora O, Tamir A, Shahar E, Sujov P. Congenital subependymal pseudocysts: own data and meta-analysis of the literature. Isr Med Assoc J. 2001;3(3):178-183.

27. Fickenscher K, Bailey Z, Saettele M, Dahl A, Lowe L. Pediatric Cranial Ultrasound: Techniques, Variants and Pitfalls. In: Bright P, editor. Neuroimaging - Methods. InTech; 2012:217-232.

28. Middleton WD, Kurtz AB, Hertzbert BS. Ultrasound: the requisites. 2nd ed. St Louis: Mosby-Year Book; 2009.

29. Bernard JP, Moscoso G, Renier D, Ville Y. Cystic malformations of the posterior fossa. Prenat Diagn. 2001;21:1064-1069.

30. Bowerman RA. Tangential sulcal echoes: potential pitfall in the diagnosis of parenchymal lesions on cranial sonography. JUltrasound Med. 1987;6:685-689.

31. Franco A, Lewis KN, Maresky H, Shelef I. Increased echogenicity of the interhemispheric sulcus: a potential diagnostic pitfall on cranial ultrasound of the newborn. Isr Med Assoc J. 2012;14(6):403-404.

32. Grant EG, Schellinger D, Richardson JD, Coffey ML, Smirniotopoulous JG. Echogenic periventricular halo: normal sonographic finding or neonatal cerebral hemorrhage. AJR Am J Roentgenol. 1983;140(4): 793-796.

33. Lowe LH, Bailey Z. State-of-the-art cranial sonography: Part 2, pitfalls and variants. AJR Am J Roentgenol. 2011;196(5):1034-1039.

34. Towbin A. Central nervous system damage in the human fetus and newborn infant. Mechanical and hypoxic injury incurred in the fetalneonatal period. Am J Dis Child. 1970;119(6):529-542.

35. Burstein J, Papile LA, Burstein R. Intraventricular hemorrhage and hydrocephalus in premature newborns: a prospective study with CT. AJR Am J Roentgenol. 1979;132(4):631-635.

36. Volpe JJ. Neurology of the Newborn. 5th ed. Philadelphia: Saunders Elsevier; 2008.

37. Volpe JJ. Neurobiology of periventricular leukomalacia in the premature infant. Pediatr Res. 2001;50:553-562.

38. de Vries LS, Eken P, Dubowitz LM. The spectrum of leukomalacia using cranial ultrasound. Behav Brain Res. 1992;49(1):1-6. 
39. Epelman M, Daneman A, Blaser SI, et al. Differential diagnosis of intracranial cystic lesions at head US: correlation with CT and MR imaging. Radiographics. 2006;26(1):173-196.

40. Resch B, Vollaard E, Maurer U, Haas J, Rosegger H, Muller W. Risk factors and determinants of neurodevelopmental outcome in cystic periventricular leucomalacia. Eur J Pediatr. 2000;159:663-670.

41. Blumenthal I. Periventricular leucomalacia: a review. Eur J Pediatr. 2004; 163:435-442.

42. Coley BD, Hogan MJ. Cystic periventricular leukomalacia of the corpus callosum. Pediatr Radiol. 1997;27:583-585.

43. Larroche JC, Amiel C. Thrombosis of the sylvian artery during the neonatal period. Anatomical study and pathogenic discussion of socalled congenital hemiplegia. Arch Fr Pediatr. 1966;23(3):257-274.

44. Mannino FL, Trauner DA. Stroke in neonates. J Pediatr. 1983;102(4): 605-610.

45. Wenghoefer M, Ettema AM, Sina F, et al. Prenatal ultrasound diagnosis in 51 cases of holoprosencephaly: craniofacial anatomy, associated malformations, and genetics. Cleft Palate Craniofac J. 2010;47(1): $15-21$.

46. Dubourg C, Bendavid C, Pasquier L, Henry C, Odent S, David V. Holoprosencephaly. Orphanet J Rare Dis. 2007;2:8.

47. Fiske CE, Filly RA. Ultrasound evaluation of the normal and abnormal fetal neural axis. Radiol Clin N Am. 1982;20:285-296.

48. Manelfe C, Sevely A. Neuroradiological study of holoprosencephalies. J Neuroradiol. 1982;9:15-45.

49. Babcock DS. The normal, absent, and abnormal corpus callosum: sonographic findings. Radiology. 1984;151(2):449-453.

50. Di Salvo DN. A new view of the neonatal brain: clinical utility of supplemental neurologic US imaging windows. Radiographics. 2001;21(4): 943-955.

51. McLeary RD, Kuhns LR, Barr M Jr. Ultrasonography of the fetal cerebellum. Radiology. 1984;151(2):439-442.

52. Wilson ME, Lindsay DJ, Levi CS, Ackerman TE, Gordon WL. US case of the day. Dandy-Walker variant with agenesis of the corpus callosum. Radiographics. 1994;14(3):678-681.

53. Goodwin L, Quisling RG. The neonatal cisterna magna: ultrasonic evaluation. Radiology. 1983;149(3):691-695.

54. McLean G, Coombs P, Sehgal A, et al. Measurement of the lateral ventricles in the neonatal head: comparison of 2-D and 3-D techniques. Ultrasound Med Biol. 2012;38(12):2051-2057.

55. Mondal P, Mukhopadhyay J, Sural S, et al. A robust method for ventriculomegaly detection from neonatal brain ultrasound images. J Med Syst. 2012;36(5):2817-2828.

56. Davies MW, Swaminathan M, Chuang SL, Betheras FR. Reference ranges for the linear dimensions of the intracranial ventricles in preterm neonates. Arch Dis Child Fetal Neonatal Ed. 2000;82(3):F218-F223.

57. Brouwer MJ, de Vries LS, Groenendaal F, et al. New reference values for the neonatal cerebral ventricles. Radiology. 2012;262(1):224-233.

58. Anderson NG, Laurent I, Cook N, Woodward L, Inder TE. Growth rate of corpus callosum in very premature infants. AJNR Am J Neuroradiol. 2005;26(10):2685-2690.
59. Armstrong RK, Fox LM, Cheong JL, Davis PG, Rogerson SK. Postnatal ultrasound reliability in cerebellar vermis assessment. Arch Dis Child Fetal Neonatal Ed. 2012;97(4):F307-F309.

60. Ozer A, Ozaksit G, Kanat-Pektas M, Ozer S. First trimester examination of fetal nasal bone in the Turkish population. J Obstet Gynaecol Res. 2010;36(4):739-744.

61. Boxma A, Lequin M, Ramenghi LA, Kros M, Govaert P. Sonographic detection of the optic radiation. Acta Paediatr. 2005;94(10): 1455-1461.

62. Armstrong DL, Bagnall C, Harding JE, Teele RL. Measurement of the subarachnoid space by ultrasound in preterm infants. Arch Dis Child Fetal Neonatal Ed. 2002;86(2):F124-F126.

63. Davies MW, Swaminathan M, Betheras FR. Measurement of the transverse cerebellar diameter in preterm neonates and its use in assessment of gestational age. Australas Radiol. 2001;45(3):309-312.

64. Wu YC, Hsieh WS, Hsu CH, et al. Relationship of neonatal cerebral blood flow velocity asymmetry with early motor, cognitive and language development in term infants. Ultrasound Med Biol. 2013;39(5): 797-803.

65. Vicenzini E, Pulitano P, Cicchetti R, et al. Transcranial Doppler for brain death in infants: the role of the fontanelles. Eur Neurol. 2010;63(3): 164-169.

66. Meyberg-Solomayer GC, Soen M, Speer R, et al. Pathological prenatal Doppler sonography findings and their association with neonatal cranial ultrasound abnormalities in a high risk collective. Ultrasound Med Biol. 2008;34(8):1193-1199.

67. Horsch S, Skiöld B, Hallberg B, et al. Cranial ultrasound and MRI at term age in extremely preterm infants. Arch Dis Child Fetal Neonatal Ed. 2010;95(5):F310-F314.

68. Maalouf EF, Duggan PJ, Counsell SJ, et al. Comparison of findings on cranial ultrasound and magnetic resonance imaging in preterm infants. Pediatrics. 2001;107(4):719-727.

69. Leijser LM, de Bruïne FT, Steggerda SJ, van der Grond J, Walther FJ, van Wezel-Meijler G. Brain imaging findings in very preterm infants throughout the neonatal period: part I. Incidences and evolution of lesions, comparison between ultrasound and MRI. Early Hum Dev. 2009;85(2):101-109.

70. Mirmiran M, Barnes PD, Keller K, et al. Neonatal brain magnetic resonance imaging before discharge is better than serial cranial ultrasound in predicting cerebral palsy in very low birth weight preterm infants. Pediatrics. 2004;114(4):992-998.

71. de Vries LS, van Haastert IC, Benders MJ, Groenendaal F. Myth: cerebral palsy cannot be predicted by neonatal brain imaging. Semin Fetal Neonatal Med. 2011;16(5):279-287.

72. Taylor GA. Recent advances in neonatal cranial ultrasound and Doppler techniques. Clin Perinatol. 1997;24(3):677-691.

73. Steggerda SJ, Leijser LM, Walther FJ, van Wezel-Meijler G. Neonatal cranial ultrasonography: how to optimize its performance. Early Hum Dev. 2009;85(2):93-99.

74. van Wezel-Meijler G, Steggerda SJ, Leijser LM. Cranial ultrasonography in neonates: role and limitations. Semin Perinatol. 2010;34(1):28-38.
Reports in Medical Imaging

\section{Publish your work in this journal}

Reports in Medical Imaging is an international, peer-reviewed, open access journal publishing original research, reports, reviews and commentaries on all areas of medical imaging. The manuscript management system is completely online and includes a very quick and fair peer-review system, which is all easy to use.

\section{Dovepress}

Visit http://www.dovepress.com/testimonials.php to read real quotes from published authors. 